\title{
Improved Detection Methods for MIMO-OFDM-CDM Communication Systems
}

\author{
Jaime Adeane, Miguel R.D. Rodrigues, Inaki Berenguer, and Ian J. Wassell \\ Laboratory for Communication Engineering \\ Department of Engineering University of Cambridge \\ 15 JJ Thomson Avenue, Cambridge CB3 0FD, United Kingdom \\ $\{$ ja316, mrdr3, ib226, ijw24\}@cam.ac.uk
}

\begin{abstract}
In this paper, we consider multiple input multiple output-orthogonal frequency division multiplexing-code division multiplexing (MIMO-OFDM-CDM) techniques to efficiently improve the link reliability/spectral efficiency of very high data rate communication systems. In particular, we apply MIMO detection methods based on lattice reduction, partial decision feedback (PDF), and BLAST ordering techniques to MIMO-OFDM-CDM systems. Simulation results show that the proposed receivers significantly outperform the conventional zero-forcing (ZF), minimum mean squared error (MMSE), and Vertical Bell Labs Layered Space Time (V-BLAST) receivers without severely compromising system complexity.

Keywords: MIMO, OFDM, CDM, lattice reduction,
\end{abstract} PDF, V-BLAST.

\section{INTRODUCTION}

The major challenges in future wireless communications system design are increased spectral efficiency and improved link reliability. The radio channel constitutes a hostile propagation medium, which suffers from fading and interference from other users. The use of multiple antennas at both ends of a wireless link promises significant improvements in terms of spectral efficiency and/or link reliability. Multiple-input multiple-output (MIMO) technology has recently become very popular since it can improve link reliability without sacrificing bandwidth efficiency.

In a very high data rate MIMO communication system, the radio channel introduces severe intersymbol interference. In this case, single-carrier based MIMO systems require highly complex equalization techniques such as a vector-maximum likelihood sequence estimator (MLSE) or a multichannel equalizer. However, multicarrier based MIMO systems, e.g., MIMO-OFDM, permit simple equalisation processes by turning the frequency selective fading channel into a set of parallel flat fading channels. Essentially, the equalisation in a MIMO-OFDM system is done by inverting a constant matrix for each OFDM tone. In order to exploit further the frequency diversity offered by radio channels, we can use either coding or alternatively, code division multiplexing (CDM) techniques in conjunction with MIMO-OFDM. The focus of this paper is on the application of CDM techniques to MIMO-OFDM systems.

In MIMO-OFDM-CDM communications systems, interference arises from the transmission of multiple signals as well as spreading code interference. These sources of interference complicate the detection process and therefore, a number of linear and non-linear detection methods have been proposed in the past. Maximum likelihood
(ML) detection is optimal but highly complex. Linear detectors such as zero-forcing (ZF) and minimum mean squared error (MMSE) as well as the non-linear detector $\mathrm{V}$-BLAST are less complex, however, their performance is inferior than that of ML [1]. In this paper, we propose two classes of improved non-linear detection schemes with a good performance-complexity tradeoff.

The first class is based on lattice reduction aided (LRA) receivers, which achieve near ML detector performance with low complexity. In particular, we extend the LRA receiver technique proposed in [2] for MIMO systems and apply it to MIMO-OFDM-CDM systems. LRA receivers with BLAST nulling, cancellation, and ordering are also presented. The second class of detection methods is based on partial decision feedback (PDF) receivers. We extend the idea proposed in [4] for MIMO systems and apply it to MIMO-OFDM-CDM systems. We also propose a combination between the two classes, i.e., hybrid LRA-PDF detectors. Finally, we compare the performance of the proposed receivers to that of several popular receiver including ZF, MMSE, and V-BLAST.

\section{System Model}

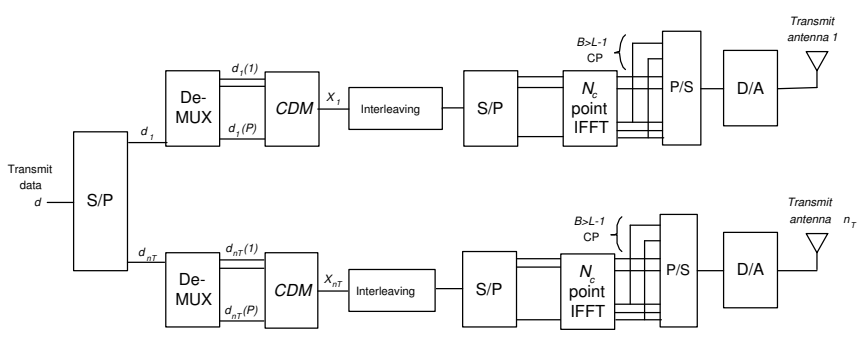

Fig. 1. MIMO-OFDM-CDM transmitter.

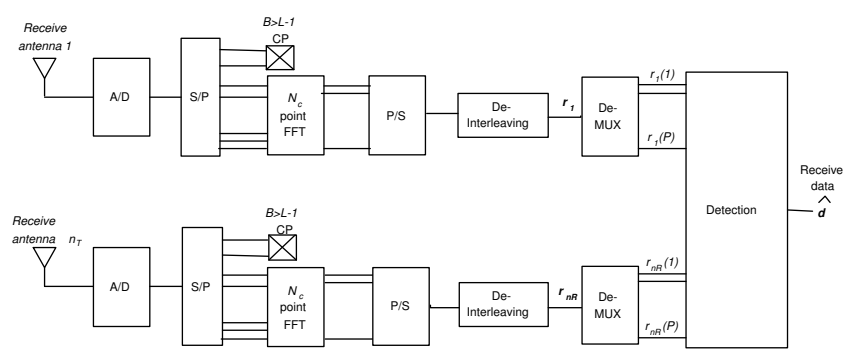

Fig. 2. MIMO-OFDM-CDM receiver. 
Figs. 1 and 2 show the block diagrams of an $n_{T} \times n_{R}$ MIMO-OFDM-CDM system, where $n_{T}$ is the number of transmit antennas and $n_{R}$ is the number of receive antennas. Initially, a block of transmit data symbols $\boldsymbol{d}$ of length $n_{T} \times P$ is split into $n_{T}$ sub-blocks of transmit data $\boldsymbol{d}_{i}$, $i=1, \ldots, n_{T}$, of length $P$, where $\mathrm{P}$ is the length of the spreading codes. Subsequently, each sub-block of transmit data $\boldsymbol{d}_{i}$ is demultiplexed into its $P$ constituent data elements $d_{i}(p), p=1, \ldots, P, i=1, \ldots, n_{T}$ and spread. In this case, the output $\boldsymbol{x}_{i}=\left[x_{i}(1), x_{i}(2), \ldots, x_{i}(P)\right]^{T}$ and the input $\boldsymbol{d}_{i}=\left[d_{i}(1), d_{i}(2), \ldots, d_{i}(P)\right]^{T}, i=1, \ldots, P$ of the CDM block of the $i$-th transmit antenna are related as follows

$$
\boldsymbol{x}_{i}=\boldsymbol{C}_{P} \boldsymbol{d}_{i},
$$

where $\boldsymbol{C}_{P}$ is a $P \times P$ CDM transform matrix, in which the columns correspond to spreading code sequences. In this paper, we consider the Hadamard transformation matrix

$$
\boldsymbol{C}_{P}=\left[\begin{array}{cc}
\boldsymbol{C}_{P / 2} & \boldsymbol{C}_{P / 2} \\
\boldsymbol{C}_{P / 2} & -\boldsymbol{C}_{P / 2}
\end{array}\right], \quad \forall P=2^{m}, m \geq 1, \boldsymbol{C}_{1}=1 .
$$

Essentially, the output of the $i$-th CDM block $\boldsymbol{x}_{i}$ is a linear combination of the data vector $\boldsymbol{d}_{i}, i=1, \ldots, n_{T}$. For each antenna, $N_{c} / P$ input data symbol blocks (each having $P$ symbols) are processed per OFDM symbol. Note that $N_{c}$ is the number of OFDM subcarriers. The resulting $N_{c} / P$ spread blocks (each having $P$ symbols) are interleaved before being input to the serial-to-parallel converter. Finally, the output of each of the $n_{T}$ serial-to-parallel converters are OFDM modulated, digital-to-analog converted, and transmitted by $n_{T}$ transmit antennas. Note that a cyclic prefix (CP) of length $B$, which is longer than the maximum delay spread of the radio channel $L$, is inserted between consecutive OFDM symbols to avoid intersymbol interference (ISI) and interchannel interference (ICI).

The receiver performs the reverse operations of the transmitter. Essentially, the input to the $j$-th demultiplexer block at the receiver, $\boldsymbol{r}_{j}=\left[r_{j}(1), r_{j}(2), \ldots, r_{j}(P)\right]^{T}, j=$ $1, \ldots, n_{R}$ is related to the inputs of the $i$-th demultiplexer block at the transmitter, $\boldsymbol{d}_{i}=\left[d_{i}(1), d_{i}(2), \ldots, d_{i}(P)\right]^{T}$, $i=1, \ldots, P$ as follows

$$
\boldsymbol{r}_{j}=\sum_{i=1}^{n_{T}} \boldsymbol{H}_{j i} \boldsymbol{C} \boldsymbol{d}_{i}+\boldsymbol{w}_{j},
$$

where the $P \times P$ diagonal matrix $\boldsymbol{H}_{j i}$ represents the fading between the $i$-th transmit antenna and $j$-th receive antenna on the $P$ subchannels where the data elements of $\boldsymbol{d}_{i}$ have been transmitted on, and $\boldsymbol{w}_{j}=\left[w_{j}(1), w_{j}(2), \ldots, w_{j}(P)\right]^{T}$ is the noise at the $j$-th receive antenna across $P$ subchannels. The additive noise $w_{j}(p), j=1, \ldots, n_{R}, p=1, \ldots, P$ is assumed to be an independent and identically distributed complex Gaussian with zero mean and complex variance of $\sigma_{w}^{2}$, so that $E\left[\boldsymbol{w}_{j} \boldsymbol{w}_{j}^{H}\right]=\sigma_{w}^{2} \boldsymbol{I}$, where $\boldsymbol{w}_{j}^{H}$ denotes the complex conjugate transpose of $\boldsymbol{w}_{j}$. Further, we assume that the input data is uncorrelated and belongs to the same unit-energy alphabet, i.e., $E\left[\boldsymbol{d}_{i} \boldsymbol{d}_{i}^{H}\right]=\boldsymbol{I}, i=1, \ldots, n_{T}$.
We can relate the block of the received data $\boldsymbol{r}=$ $\left[\boldsymbol{r}_{1}^{T}, \boldsymbol{r}_{2}^{T}, \ldots, \boldsymbol{r}_{n_{R}}^{T}\right]^{T}$, which is the collection of inputs to the receiver demultiplexers, to the block of transmit data $\boldsymbol{d}=\left[\boldsymbol{d}_{1}^{T}, \boldsymbol{d}_{2}^{T}, \ldots, \boldsymbol{d}_{n_{T}}^{T}\right]^{T}$ as follows

$$
\boldsymbol{r}=\mathcal{H} \boldsymbol{d}+\boldsymbol{w}
$$

where $\boldsymbol{w}=\left[\boldsymbol{w}_{1}^{T}, \boldsymbol{w}_{2}^{T}, \ldots, \boldsymbol{w}_{n_{R}}^{T}\right]^{T}$ and

$$
\mathcal{H}=\left[\begin{array}{ccc}
\boldsymbol{H}_{11} \boldsymbol{C} & \ldots & \boldsymbol{H}_{1 n_{T}} \boldsymbol{C} \\
\vdots & \ddots & \vdots \\
\boldsymbol{H}_{n_{R} 1} \boldsymbol{C} & \ldots & \boldsymbol{H}_{n_{R} n_{T}} \boldsymbol{C}
\end{array}\right]
$$

We define the $\left(n_{R} P\right) \times\left(n_{T} P\right)$ matrix $\mathcal{H}$ as the extended MIMO-OFDM-CDM channel matrix.

\section{Detection Methods for A MIMO-OFDM-CDM SYSTEM}

In the MIMO-OFDM-CDM system, shown in Figs. 1 and 2, data symbols are spread and multiplexed over multiple subcarriers using orthogonal codes to obtain further frequency diversity in frequency-selective fading channels. Theoretically, orthogonal spreading codes will minimize self-interference between multiple data symbols. However, in fading channels, the orthogonality between spreading codes is destroyed due to different channel gains at different subcarriers. It is therefore of paramount importance to devise efficient detection methods to combat selfinterference as well as to utilize the potential diversity in MIMO-OFDM-CDM. We will review some basic receivers, including ZF, MMSE, and V-BLAST before elaborating on the proposed detection methods.

\section{A. Basic Linear Receivers}

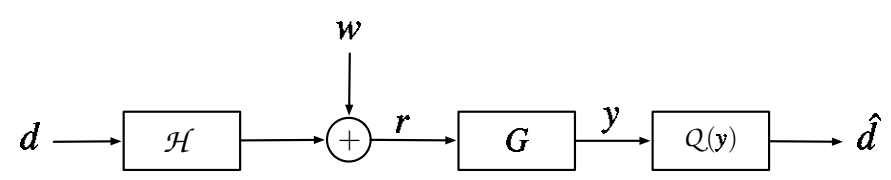

Fig. 3. Traditional linear receiver.

Let us consider the received signal vector in (4). A linear receiver will transform the received unequalised signal $\boldsymbol{r}$ as follows

$$
\boldsymbol{y}=\boldsymbol{G r}=\boldsymbol{G H} \boldsymbol{H}+\boldsymbol{G w},
$$

where $\boldsymbol{G}$ is the matrix equalizer. The estimate of the transmitted data vector is $\hat{\boldsymbol{d}}=\mathcal{Q}(\boldsymbol{y})$. This process is shown in Fig. 3. For ZF equalization, $\boldsymbol{G}=\mathcal{H}^{\dagger}$, where $\mathcal{H}^{\dagger}=\left(\mathcal{H}^{H} \mathcal{H}\right)^{-1} \mathcal{H}^{H}$ is the pseudoinverse of the channel matrix $\mathcal{H}$. The ZF criterion suffers from noise enhancement especially if $\mathcal{H}$ is rank-deficient or ill-conditioned. On the other hand, for MMSE linear equalization, $\boldsymbol{G}=$ $\sigma_{d}^{2} \mathcal{H}^{H}\left(\mathcal{H} \mathcal{H}^{H}+\sigma_{w}^{2} \boldsymbol{I}_{n_{R}}\right)^{-1}$. The MMSE criterion minimizes the error due to both the noise and interference. There are also nonlinear receivers that consist of nulling and cancellation operations in an optimal order, such as 
BLAST receivers [5]. They offer better performance than linear receivers with a moderate increase in complexity. However, the performance of these receivers are far away from that of the much more complex ML receivers.

\section{B. Lattice-Reduction-Aided (LRA) Receivers}

The LRA receiver technique that is first proposed in [2], is applicable for $2 \times 2$ MIMO systems. In [3], the algorithm was further extended to a general $n_{R} \times n_{T}$ MIMOOFDM system, where $n_{R} \geq n_{T}$. In this paper, we apply the technique proposed in [3] to MIMO-OFDM-CDM systems. An $n_{R} \times n_{T}$ MIMO-OFDM-CDM channel matrix $\mathcal{H}$

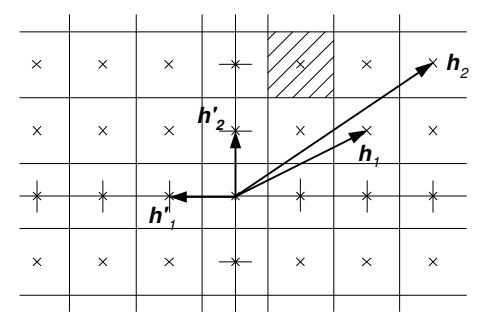

Fig. 4. Original and new bases.

is characterized by its columns, $\boldsymbol{h}_{i}, i=1, \ldots, n_{T} P$, which are the basis of $\mathcal{H}$. As shown in Fig. 4, if the angle between 2 basis vectors represented by 2 columns of $\mathcal{H}$, e.g. $\boldsymbol{h}_{1}$ and $\boldsymbol{h}_{2}$, is very narrow (i.e., the vectors are correlated), a small amount of noise can make a received symbol fall out of its own decision region and cause the decoder to make a wrong decision [2]. Therefore, the objective of the LRA technique [3] is to find a change of basis, $\boldsymbol{F}$ which transforms $\mathcal{H}$ into $\mathcal{H}^{\prime}$ such that the decision regions for a specific lattice and decoder are optimized. An algorithm to compute the change of basis $\boldsymbol{F}$, for arbitrary numbers of transmit and receive antennas with $n_{R} \geq n_{T}$, is given in [3].

Assuming the received vector $\boldsymbol{r}$ in Fig. 3 is already represented in the new reduced basis $\mathcal{H}^{\prime}$,

$$
\boldsymbol{r}^{\prime}=\mathcal{H}^{\prime} \boldsymbol{z}+\boldsymbol{n},
$$

where $\boldsymbol{z}$ is the transmitted symbols represented in the new basis, i.e., $\boldsymbol{z}=\boldsymbol{F}^{-1} \boldsymbol{d}$. Since $\mathcal{H}^{\prime}=\mathcal{H} \boldsymbol{F}$, for a ZF receiver where $\boldsymbol{G}=(\mathcal{H} \boldsymbol{F})^{\dagger}$, (6) can be written as

$$
\boldsymbol{y}=\boldsymbol{G} \mathcal{H} \boldsymbol{F} \boldsymbol{z}+\boldsymbol{G n} .
$$

The estimate of $\boldsymbol{z}$ is $\hat{\boldsymbol{z}}=\mathcal{Q}(\boldsymbol{y})$. With this LRA technique, the quantization consists of a rounding operation whereby the real and imaginary parts are rounded separately. Finally, $\hat{z}$ is transformed to its original basis by performing the operation $\hat{\boldsymbol{d}}=\boldsymbol{F} \hat{\boldsymbol{z}}$.

To use the lattice theory and the decoding operation in (8), the original points in the constellation are required to consist of symbols in $\mathbb{Z}_{\mathbb{C}}$. In this paper, we consider $M$-QAM constellations such that $\Re\{d\} \in\{-\sqrt{M}+$ $1, \ldots, \sqrt{M}-1\}$ and $\Im\{d\} \in\{-\sqrt{M}+1, \ldots, \sqrt{M}-1\}$, thus, to convert the symbols into contiguous integers, one option is to shift the original constellation by $\boldsymbol{l}=[1+i, \ldots, 1+i]^{T}$ and scale it by $1 / 2[3]$.
To summarise, with LRA receivers, we firstly reduce the basis of the MIMO-OFDM-CDM channel matrix $\mathcal{H}$ to a new basis representing the same lattice in which its column vectors are less correlated. Secondly, we decode the symbols in the new basis, and finally transform the decoded symbols into the original basis. All of these operations are performed at the receiver. Therefore, the operations at the receiver consist of two steps [3]: (a) scaling, shifting, and equalizing in the new basis

$$
\boldsymbol{y}=\underbrace{(\mathcal{H} \boldsymbol{F})^{\dagger}}_{\text {equalize in new basis }} \underbrace{\frac{1}{2}}_{\text {scale }}[\underbrace{\mathcal{H} \boldsymbol{d}+\boldsymbol{n}}_{\text {rx signal-y }}+\underbrace{\mathcal{H} \boldsymbol{l}}_{\text {shift }}]
$$

and (b) slicing, returning to the original basis, and undoing the scaling and shifting as

$$
\hat{\boldsymbol{d}}=2 \boldsymbol{F} \mathcal{Q}(\boldsymbol{y})-\boldsymbol{l} .
$$

As a special case, when $n_{T}=n_{R}$ and the channel is of full rank, (9) can be written as

$$
\boldsymbol{y}=\boldsymbol{F}^{-1} \frac{1}{2}(\boldsymbol{d}+\boldsymbol{l})+\frac{1}{2} \boldsymbol{G} \boldsymbol{w} .
$$

\section{V-BLAST Receiver}

In a pioneering work, Golden et al. [5] provided a multiantenna prototype that achieves a very high spectral efficiency by the use of the V-BLAST detection algorithm which realizes a good tradeoff between complexity and performance. In [7], Hassibi proposes an improvement over the original V-BLAST algorithm in [5], by performing the nulling operation using unitary transformations. This approach results in a lower complexity algorithm and better numerical stability. In [8], Damen et. al proposed an iterative detection algorithm for MIMO systems to further improve the detector performance in [7] while reducing the computational complexity. The approach was based on two QR decompositions of the channel transfer matrix which are then used iteratively to perform hard or soft intersubstream interference cancellation. In this work we extend the improved BLAST detection algorithm in [5], [8], which are applicable to MIMO systems, to MIMO-OFDM-CDM systems. We then use the V-BLAST cancellation and ordering technique, and integrate it in the lattice reduction algorithm.

To summarise, the steps are as follows: First, we reduce the basis of the extended channel matrix $\mathcal{H}$ and calculate the change of basis, $\boldsymbol{F}$ according to the lattice reduction algorithm as detailed in [3]. Second, by using the V-BLAST cancelling and optimal ordering technique, we perform the detection in the new basis. Finally, the detected symbols are transformed to the original basis.

\section{Partial Decision Feedback Receiver}

The noise predictive decision-feedback (DF) detector is a non-linear detection strategy originally formulated for MIMO systems. It can significantly outperform a linear detector, provided that the inputs are detected using the 
so-called BLAST ordering [4]. This approach is called ordered DF detector (ODF). The important advantage of the noise-predictive detector is that it only requires the knowledge of the autocorrelation matrix of the noise, and not the channel. The DF detector requires an order of magnitude more computations than the linear detector, and therefore, Waters [4] proposes a partial decision-feedback detector which is a combination of the ordered DF detector (ODF) and the linear detector, applicable for MIMO systems. Using a low-complexity implementation based on the noise-predictive view of the DF detector, PDF was shown to perform within $0.3 \mathrm{~dB}$ of the BLAST ordered DF detector in MIMO systems, while requiring $38 \%$ of its computations. The technique used is based on noise prediction decision feedback. In this paper, we apply the algorithm proposed in [4] to MIMO-OFDM-CDM systems.

Referring to Fig. 3, PDF detection begins with zeroforcing (ZF) linear detection

$$
\boldsymbol{y}=\boldsymbol{G r}=\boldsymbol{G} \mathcal{H} \boldsymbol{d}+\boldsymbol{G w}
$$

where $\boldsymbol{G}=\boldsymbol{H}^{\dagger}$. Eq.(12) can be rewritten as

$$
\boldsymbol{y}=\boldsymbol{d}+\boldsymbol{n},
$$

where $\boldsymbol{y}=\left[\boldsymbol{y}_{1}^{T}, \boldsymbol{y}_{2}^{T}, \ldots, \boldsymbol{y}_{n_{R}}^{T}\right]^{T}$ and the noise $\boldsymbol{n}=$ $\left[\boldsymbol{n}_{1}^{T}, \boldsymbol{n}_{2}^{T}, \ldots, \boldsymbol{n}_{R}^{T}\right]$ has autocorrelation matrix $E\left[\boldsymbol{n}(\boldsymbol{n})^{H}\right]=$ $\sigma_{n}^{2}\left((\mathcal{H})^{H} \mathcal{H}\right)^{-1}$. Note that $\sigma_{n}^{2}$ is the noise variance.

Based upon the output of the linear detector, we detect and quantize the symbol with the smallest MSE. It can be shown that the MSE of the first symbol is proportional to the squared norm of the corresponding row of the channel pseudoinverse and therefore, the index of the first detected symbol is [4]

$$
i_{1}=\operatorname{argmin}_{\mathrm{j} \in\left\{1, \ldots, \mathrm{n}_{\mathrm{T}}\right\}}\left\|\boldsymbol{g}_{\mathrm{j}}\right\|^{2},
$$

where $\boldsymbol{g}_{j}$ is the $j$-th row of $\boldsymbol{G}$.

The first detected symbol that has the smallest MSE, $\hat{d}_{i_{1}}$, is found by quantizing $y_{i_{1}}$ directly. For other symbols, the PDF detector applies linear prediction to reduce the noise variance before quantizing. Note that based on the detection of the symbol having the smallest MSE, $\hat{d}_{i_{1}}$, the receiver deduces the $i_{1}$-th noise sample,

$$
n_{i_{1}}=y_{i_{1}}-\hat{d}_{i_{1}} .
$$

Based on the correlation between noise samples, by knowing $n_{i_{1}}$, we can predict $\left\{n_{i_{v}}\right\}$ for $v>1$. Let $w_{v}\left(y_{i_{1}}-\hat{d}_{i_{1}}\right)$ for some constants $w_{v}$ denote the predicted value of $n_{i_{v}}$ based on $n_{i_{1}}$. The PDF detector subtracts this predicted noise sample from $y_{i_{v}}$, and therefore when the symbol with the smallest MSE is detected correctly, the noise variance for the $i_{v}$-th symbol is reduced to [4]

$$
\begin{aligned}
E\left[\left|n_{i_{v}}-w_{v} n_{i_{1}}\right|^{2}\right] & =E\left[\left|\boldsymbol{g}_{i_{v}} \boldsymbol{w}\right|^{2}\right] \\
& =\sigma_{n}^{2}\left\|\boldsymbol{g}_{i_{v}}-w_{v} \boldsymbol{g}_{i_{1}}\right\|^{2} .
\end{aligned}
$$

When $w_{v}$ is chosen to minimize the noise variance, the term $w_{v} \boldsymbol{g}_{i_{1}}$ reduces to the projection of $\boldsymbol{g}_{i_{v}}$ onto the subspace spanned by $\boldsymbol{g}_{i_{1}}$. This leads to a simple equation for finding the prediction coefficients [4]

$$
w_{v}=\boldsymbol{g}_{i_{v}}\left(\boldsymbol{g}_{i_{1}}\right)^{H}\left\|g_{i_{1}}\right\|^{2} .
$$

The $v$-th symbol is detected according to

$$
\hat{d}_{i_{v}}=\mathcal{Q}\left\{y_{i_{v}}-w_{v}\left(y_{i_{1}}-\hat{d}_{i_{1}}\right)\right\},
$$

where $\mathcal{Q}($.$) denotes the quantization operation.$

\section{E. Hybrid LRA-PDF Receivers}

Based on the two techniques, LRA and PDF, we propose a combination between the two, namely hybrid LRA-PDF. With the hybrid LRA-PDF technique, the extended channel matrix $\mathcal{H}$ is first reduced to a new basis $\mathcal{H}^{\prime}$. After ZF detection in the new basis $\mathcal{H}^{\prime}$, we determine the index, $t$, of the symbol with the smallest MSE. After calculating the received symbol at position $t$, and making a decision on it in the reduced basis, the linear prediction steps as is described in the previous section follow. Note that in the new basis, all quantization operations are based on a rounding operation. After all of the data symbols are detected in the new basis, we return to the original basis to obtain $\hat{\boldsymbol{d}}$.

The algorithm describing this new detection technique is detailed in Algorithm 1.

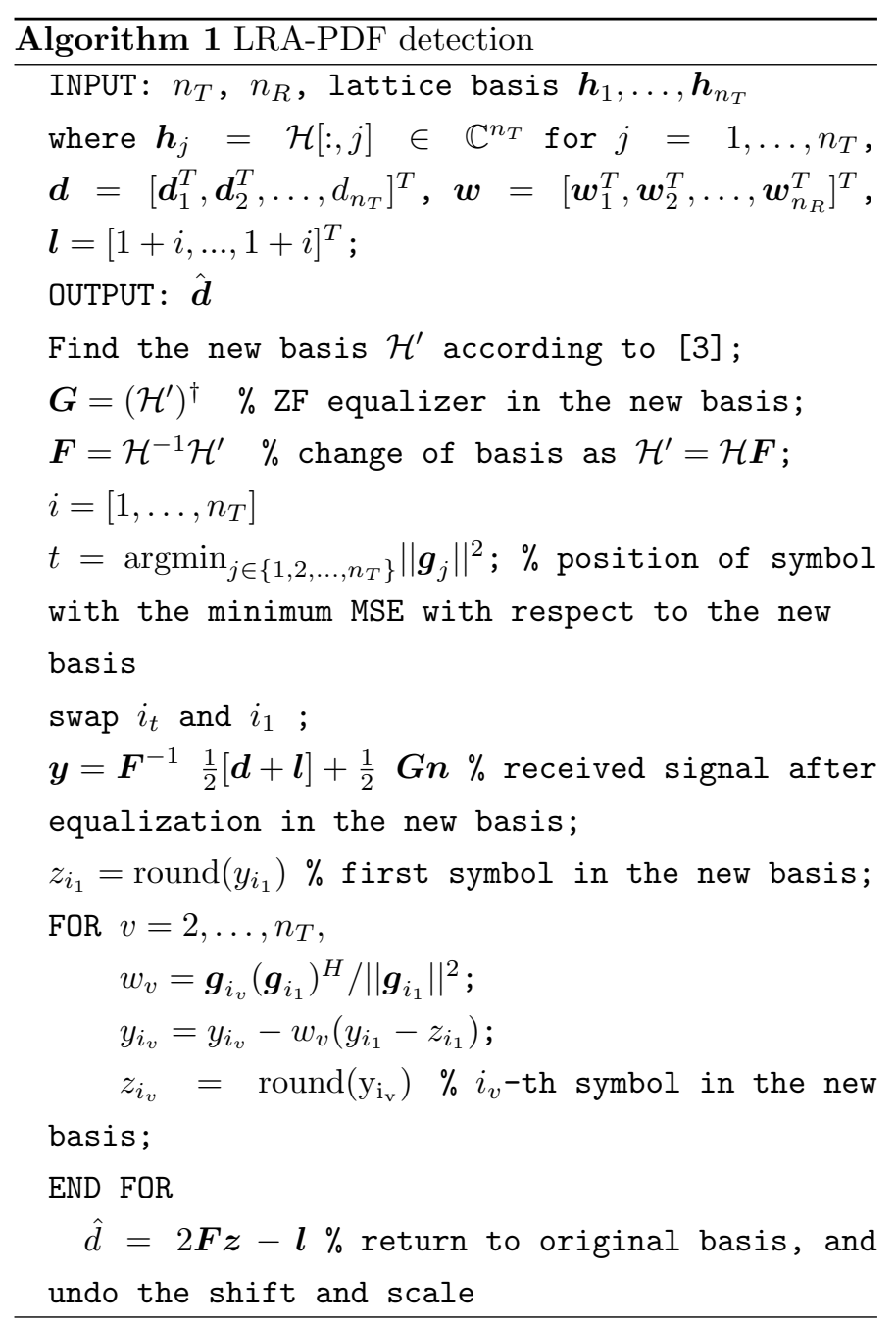




\section{Simulation Results}

The simulations presented in this section were conducted with the following parameters: Total signal bandwidth $=$ $20 \mathrm{MHz}$, number of OFDM subcarriers, $N_{c}=64, n_{T}=$ 2 transmit antennas, $n_{R}=2$ receive antennas, Hadamard code length, $P=2$ and 4 , interleaving depth, $\pi=N_{c} / P$, length of cyclic prefix, $B=16$, and QPSK modulation. The frequency-selective fading channel is a multipath channel with $L=3$ resolvable paths delayed by $0,0.4$, and $0.9 \mu \mathrm{s}$. The distribution of the multipath complex channel gains is $h_{m, v}[l] \sim \mathcal{N}_{c}\left(0, \sigma_{h, l}^{2}\right)$, for $m=1, \ldots, n_{R}$ and $v=1, \ldots, n_{T}$ with $\sigma_{h, 0}^{2}=1, \sigma_{h, 1}^{2}=0.3$, and $\sigma_{h, 2}^{2}=0.1$. Channel noise is complex Gaussian with symmetric density function $\mathcal{N}_{c}(0,1)$. The channel is assumed to be known at the receiver. BER results are averaged over 50 independent channel realizations for each SNR.

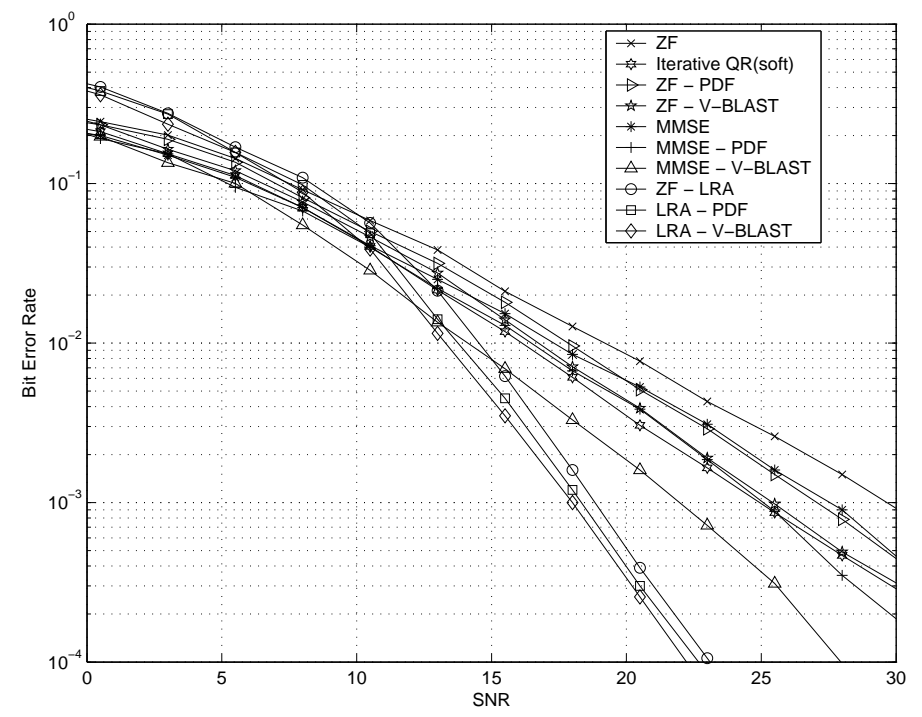

Fig. 5. BER performance of $2 \times 2$ MIMO-OFDM-CDM systems with $\mathrm{P}=2$.

Figs. 5 and 6 show simulation results for the Hadamard code of lengths $P=2$ and $P=4$, respectively. It can be seen that the proposed receiver structures are superior to the conventional ZF and MMSE linear receivers. We also note that the LRA and hybrid receivers outperform V-BLAST receivers, e.g., at $\mathrm{BER}=10^{-4}$, for $P=2$ and $P=4$, the LRA based detectors outperform MMSE - VBLAST by $6 \mathrm{~dB}$ and $9 \mathrm{~dB}$, respectively. From the slope of the graphs in both figures, it can be seen that the lattice reduction based detectors achieve the highest diversity order.

\section{Conclusion}

In this paper we have presented different suboptimum detection schemes, based on lattice reduction and noise prediction for MIMO-OFDM-CDM communications systems. Simulation results have shown that the proposed detectors provide significant BER performance improvements over the linear detectors, i.e., ZF and MMSE, and

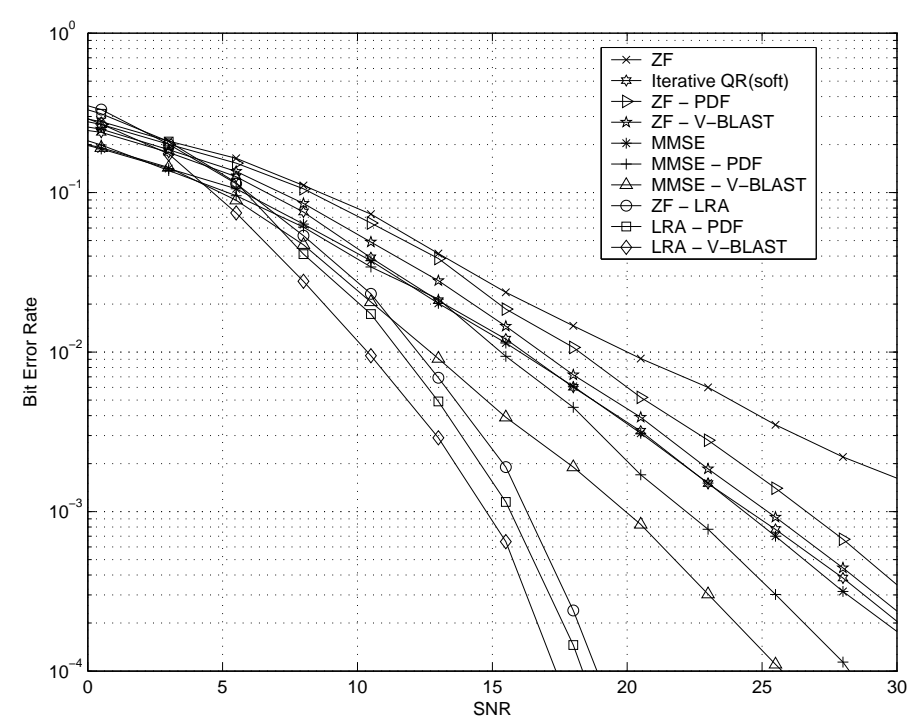

Fig. 6. BER performance of $2 \times 2$ MIMO-OFDM-CDM systems with $\mathrm{P}=4$.

the non-linear detector V-BLAST. Lattice reduction based techniques have the highest diversity order, however are slightly more complex than the others as we increase the number of antennas or the length of the spreading sequence.

\section{ACKNowledgement}

The authors would like to thank Deric W. Waters for providing the pre-print of [4].

\section{REFERENCES}

[1] K. Ha and K.B. Lee, "OFDM-CDM with V-BLAST detection and its extension to MIMO systems," in Proc. IEEE VTC, vol.1, Orlando, Florida, Apr. 2003.

[2] H. Yao and G.W. Wornell, "Lattice-reduction-aided detectors for MIMO communication systems," in Proc. IEEE Globecom, Taipei, Taiwan, Nov. 2002.

[3] I. Berenguer, J. Adeane, I.J. Wassell, and X. Wang, "LatticeReduction-Aided Receivers for MIMO-OFDM in spatial multiplexing systems," In Proc. IEEE PIMRC 2004, Barcelona, Spain, Sep. 2004 (to appear).

[4] D.W. Waters and J.R. Barry, "Partial decision-feedback detection for multiple-input multiple-output channels," In Proc. IEEE ICC 2004, Paris, France, June 2004 (to appear).

[5] G.D. Golden, G.J. Foschini, R.A. Valenzuela, and P.W. Wolniansky, "Detection algorithm and initial laboratory results using the V-BLAST space-time communication architecture," Electron. Lett., 35(1):14-15, Jan. 1999.

[6] H. Bolcskei, D. Gesbert, A.J. Pauraj. On the capacity of OFDMbased multi-antenna systems. in Proc. IEEE ICASSP, Istanbul, Turkey, June 2000.

[7] B. Hassibi, "An efficient square-root algorithm for BLAST," in Proc. IEEE ICASSP, Istanbul, Turkey, Jun. 2000.

[8] M.O. Damen, K. Abed-Meraim, and S. Burykh, "Iterative QR Detection for BLAST," Wireless Personal Communications, 19(3):179-192, Dec. 2001. 